\title{
Synthesis of (-)-Chaetominine
}

\author{
Barry B. Snider* and Xiaoxing Wu \\ Department of Chemistry MS 015, Brandeis University, Waltham, MA 02454-9110
}

\section{Supporting Material}

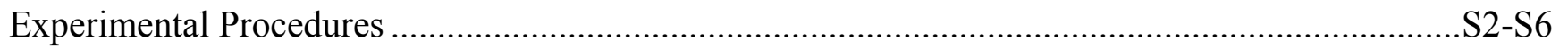

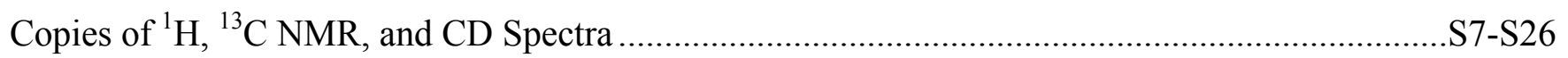


General procedures. NMR spectra were recorded at $400 \mathrm{MHz}$ in $\mathrm{CDCl}_{3}$ with $\mathrm{TMS}$ as internal standard unless otherwise indicated. Chemical shifts are reported in $\delta$, coupling constants in $\mathrm{Hz}$, and IR spectra in $\mathrm{cm}^{-1}$. Spectra in DMSO- $d_{6}$ are referenced to the residual solvent peaks at $\delta 2.50\left({ }^{1} \mathrm{H}\right)$ and $\delta 39.51\left({ }^{13} \mathrm{C}\right)$. Powered zinc was activated by washing sequentially with $3 \mathrm{M} \mathrm{HCl}$, water, EtOH, and ether and drying under reduced pressure. ${ }^{10}$

Methyl ( $\alpha R, 2 S, 9 S, 9 a R)-2,3,9,9 a-T e t r a h y d r o-2-m e t h y l-3-0 x 0-1-$ [(phenylmethoxy)carbonyl]- $\alpha-[[(2,2,2-t$ richloroethoxy)carbonyl]amino]-9[(triethylsilyl)oxy]-1H-imidazo(1,2-a)indole-9-propanoate (11). TESOTf (67 $\mu \mathrm{L}, 0.3$ mmol) was added dropwise by syringe over $5 \mathrm{~min}$ to a solution of $7(61.2 \mathrm{mg}, 0.1 \mathrm{mmol})$ and 2,6lutidine $(58 \mu \mathrm{L}, 0.5 \mathrm{mmol})$ in $\mathrm{CH}_{2} \mathrm{Cl}_{2}(5 \mathrm{~mL})$ under $\mathrm{N}_{2}$ at $0{ }^{\circ} \mathrm{C}$. The resulting solution was allowed to warm to $25^{\circ} \mathrm{C}$ and stirred for $1 \mathrm{~h}$. The solvent was removed under reduced pressure. Flash chromatography on $\mathrm{MeOH}$-deactivated silica gel $\left(50: 1 \mathrm{CH}_{2} \mathrm{Cl}_{2} / \mathrm{EtOAc}\right)$ gave $58.0 \mathrm{mg}$ (80\%) of 11 as a white solid: $\operatorname{mp} 61-65{ }^{\circ} \mathrm{C} ;[\alpha]^{22} \mathrm{D}-1.4\left(\right.$ c 3.32, $\left.\mathrm{CHCl}_{3}\right) ;{ }^{1} \mathrm{H} \mathrm{NMR} .52$ (d, $1, J=$ 7.9), 7.42-7.34 (m, 7), $7.18(\mathrm{dd}, 1, J=7.6,7.6), 6.13(\mathrm{br}, 1, \mathrm{NH}), 5.87(\mathrm{~s}, 1), 5.33$ (d, 1, J= 12.2), $5.25(\mathrm{~d}, 1, J=12.2), 4.75(\mathrm{~d}, 1, J=11.9), 4.70(\mathrm{~d}, 1, J=11.9), 4.63-4.57(\mathrm{~m}, 2), 3.56(\mathrm{~s}, 3), 2.31$ $(\mathrm{dd}, 1, J=14.7,4.3), 2.03(\mathrm{dd}, 1, J=14.7,7.3), 1.55(\mathrm{~d}, 3, J=6.7), 0.89$ (t, 9, $J=7.9), 0.64-0.51$ $(\mathrm{m}, 6) ;{ }^{13} \mathrm{C}$ NMR 171.8, 169.8, 155.3, 154.1, 137.1 (br), 136.3, 135.6, 130.1, 128.6 (2 C), 128.5, 128.3 (2 C), 126.0, 125.4, 116.7, 95.3, 86.8, 84.1, 74.7, 68.2, 59.4, 52.3, 51.0, 37.9, 18.2, 6.9 (3 C), 6.5 (3 C); IR (neat) 3345, 1739, 1732, 1717; HRMS (Qtof) calcd for $\mathrm{C}_{32} \mathrm{H}_{40} \mathrm{~N}_{3} \mathrm{O}_{8} \mathrm{NaSiCl}_{3}$ $\left(\mathrm{MNa}^{+}\right) 750.1548$, found 750.1555 .

\section{Methyl ( $\alpha R, 2 S, 9 S, 9 a S)-2,3,9,9 a-T e t r a h y d r o-2-m e t h y l-3-o x 0-\alpha-[[(2,2,2-$} trichloroethoxy)carbonyl]amino]-9-[(triethylsilyl)oxy]-1H-imidazo(1,2-a)indole-9propanoate (12). To a solution of $11(75.3 \mathrm{mg}, 103 \mu \mathrm{mol})$ in methanol (3 mL) was added 20 $\mathrm{mg}$ of $10 \% \mathrm{Pd} / \mathrm{C}$. The suspension was stirred at $25^{\circ} \mathrm{C}$ under 1 atm of $\mathrm{H}_{2}$ for $30 \mathrm{~min}$. The catalyst was filtered off and the filtrate was concentrated under reduced pressure. Flash chromatography on $\mathrm{MeOH}$-deactivated silica gel (5:1 hexanes/EtOAc) gave $34.6 \mathrm{mg}$ (56\%) of 12 as a white solid: $\operatorname{mp} 152-154{ }^{\circ} \mathrm{C} ;[\alpha]^{22}+27.5\left(\right.$ c $\left.1.13, \mathrm{CHCl}_{3}\right) ;{ }^{1} \mathrm{H}$ NMR $7.56(\mathrm{~d}, 1, J=7.6)$, 
$7.34(\mathrm{dd}, 1, J=7.6,7.6), 7.32(\mathrm{~d}, 1, J=7.6), 7.15(\mathrm{dd}, 1, J=7.6,7.6), 6.69(\mathrm{~d}, 1, J=6.1, \mathrm{NH})$,

$5.43(\mathrm{~s}, 1), 4.71(\mathrm{~d}, 1, J=12.2), 4.67(\mathrm{~d}, 1, J=12.2), 4.47-4.42(\mathrm{~m}, 1), 4.18(\mathrm{q}, 1, J=6.7), 3.69$

(s, 3), 2.74-2.56 (br, 1, NH), $2.46(\mathrm{dd}, 1, J=14.7,4.3), 2.25(\mathrm{dd}, 1, J=14.7,8.9), 1.44(\mathrm{~d}, 3, J=$ 6.7), $0.88(\mathrm{t}, 9, J=7.9), 0.56-0.40(\mathrm{~m}, 6) ;{ }^{13} \mathrm{C} \mathrm{NMR} 172.4,170.8,154.0,137.3,136.8,130.2$, $125.0,124.9,115.2,95.4,85.6,83.8,74.5,59.1,52.4,51.6,38.3,17.4,6.9$ (3 C), 6.2 (3 C); IR (neat) 3320, 1739, 1732, 1715; HRMS (Qtof) calcd for $\mathrm{C}_{24} \mathrm{H}_{35} \mathrm{~N}_{3} \mathrm{O}_{6} \mathrm{SiCl}_{3}\left(\mathrm{MH}^{+}\right.$) 594.1361, found 594.1370.

\section{$(2 S, 4 R, 5 a S, 9 c S)-4,5,5 a, 9 c-T e t r a h y d r o-2-m e t h y l-4-[[(2,2,2-$}

\section{trichloroethoxy)carbonyl]amino]-5a-[(triethylsilyl)oxy]-3H-2a,9b-}

diazacyclopenta[jk]fluorene-1,3(2H)-dione (13). A solution of amine 12 (22.6 mg, $38 \mu \mathrm{mol})$ and DMAP ( $1 \mathrm{mg}, 8 \mu \mathrm{mol})$ in dry toluene $(0.7 \mathrm{~mL})$ was heated to $115^{\circ} \mathrm{C}$ under $\mathrm{N}_{2}$ for $3 \mathrm{~d}$ in a sealed tube. The solvent was removed under reduced pressure. Flash chromatography on MeOH-deactivated silica gel (4:1 hexanes/EtOAc) gave $16.9 \mathrm{mg}(79 \%)$ of 13: $[\alpha]^{22}{ }_{\mathrm{D}}-31.4(c$ 0.32, $\left.\mathrm{CHCl}_{3}\right) ;{ }^{1} \mathrm{H}$ NMR $7.61(\mathrm{~d}, 1, J=7.6), 7.41(\mathrm{dd}, 1, J=7.6,7.6), 7.37$ (d, 1, J= 7.6), 7.22 $(\mathrm{dd}, 1, J=7.6,7.6), 6.02(\mathrm{~d}, 1, J=4.9, \mathrm{NH}), 5.37(\mathrm{~s}, 1), 4.80(\mathrm{~d}, 1, J=12.2), 4.72(\mathrm{~d}, 1, J=$ 12.2), 4.55-4.52 (m, 1), 4.43 (q, $1, J=6.7), 3.01(\mathrm{dd}, 1, J=12.5,3.4), 1.78(\mathrm{dd}, 1, J=12.5$, 12.5), $1.73(\mathrm{~d}, 3, J=6.7), 0.87(\mathrm{t}, 9, J=7.9), 0.45$ (q, $6, J=7.9) ;{ }^{13} \mathrm{C}$ NMR 172.7, 167.7, 154.0, $140.0,134.0,130.9,125.7,125.0,115.6,95.3,83.7,79.6,74.7,59.6,50.1,41.5,14.8,6.8$ (3 C), 6.0 (3 C); IR (neat) 3330, 1739, 1733, 1682; HRMS (Qtof) calcd for $\mathrm{C}_{23} \mathrm{H}_{30} \mathrm{~N}_{3} \mathrm{O}_{5} \mathrm{NaSiCl}_{3}$ $\left(\mathrm{MNa}^{+}\right)$584.0918, found 584.0941. A 1D NOESY experiment showed an NOE from the proton at $\delta$ 4.55-4.52 to the proton at $\delta 5.37$.

(2S,4R,5aS,9cS)-4-Amino-4,5,5a,9c-tetrahydro-2-methyl-5a-[(triethylsilyl)oxy]-3H2a,9b-diazacyclopenta[jk]fluorene-1,3(2H)-dione (14). To a solution of $\mathbf{1 3}$ (16.9 mg, $30 \mu \mathrm{mol})$ in $\mathrm{MeOH}(1 \mathrm{~mL})$ and $\mathrm{AcOH}(1 \mathrm{~mL})$ was added activated zinc ${ }^{10}(194 \mathrm{mg}, 3.0 \mathrm{mmol})$. The mixture was vigorously stirred at $25^{\circ} \mathrm{C}$ for $2.5 \mathrm{~h}$ and diluted with $\mathrm{CH}_{2} \mathrm{Cl}_{2}(10 \mathrm{~mL})$. Excess zinc was filtered off through a pad of Celite and the filtrate was washed with saturated $\mathrm{NaHCO}_{3}$ solution, dried $\left(\mathrm{Na}_{2} \mathrm{SO}_{4}\right)$, and concentrated to yield $13.2 \mathrm{mg}$ of crude amine 14. Flash 
chromatography on $\mathrm{MeOH}$-deactivated silica gel $\left(50: 1 \mathrm{CH}_{2} \mathrm{Cl}_{2} / \mathrm{MeOH}\right)$ yielded $10.2 \mathrm{mg}(88 \%)$ of 14: $[\alpha]^{22}{ }_{\mathrm{D}}+37.8\left(c 0.33, \mathrm{CHCl}_{3}\right) ;{ }^{1} \mathrm{H} \mathrm{NMR} 7.60$ (d, 1, J= 7.9), 7.40 (dd, 1, J = 7.9, 7.9), 7.35 $(\mathrm{d}, 1, J=7.9), 7.20(\mathrm{dd}, 1, J=7.9,7.9), 5.31(\mathrm{~s}, 1), 4.38(\mathrm{q}, 1, J=6.7), 3.71(\mathrm{dd}, 1, J=12.2$, 3.1), $2.62(\mathrm{dd}, 1, J=13.1,3.1), 1.78(\mathrm{dd}, 1, J=13.1,12.2), 1.72(\mathrm{~d}, 3, J=6.7), 0.84(\mathrm{t}, 9, J=$ 7.9), 0.46-0.35 (m, 6); ${ }^{13} \mathrm{C}$ NMR 173.3, 172.3, 140.2, 134.5, 130.7, 125.5, 124.8, 115.5, 84.0, 79.8, 59.3, 49.9, 44.0, 14.8, 6.8 (3 C), 5.9 (3 C); IR (neat) 3374, 1738, 1681; HRMS (Qtof) calcd for $\mathrm{C}_{20} \mathrm{H}_{30} \mathrm{~N}_{3} \mathrm{O}_{3} \mathrm{Si}\left(\mathrm{MH}^{+}\right)$388.2056, found 388.2049.

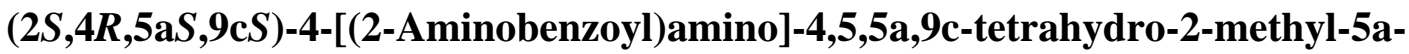
[(triethylsilyl)oxy]-3H-2a,9b-diazacyclopenta[jk]fluorene-1,3(2H)-dione (15). To a solution of $14(16.1 \mathrm{mg}, 42 \mu \mathrm{mol})$ in benzene $(5 \mathrm{~mL})$ was added isatoic anhydride $(10.2 \mathrm{mg}, 63 \mu \mathrm{mol})$ in one portion. The suspension was refluxed under $\mathrm{N}_{2}$ for $12 \mathrm{~h}$ and benzene was removed under reduced pressure. Flash chromatography on $\mathrm{MeOH}$-deactivated silica gel (6:1 hexanes/EtOAc) gave $17.3 \mathrm{mg}(82 \%)$ of 15: $[\alpha]^{22}{ }_{\mathrm{D}}-91.3\left(c\right.$ $\left.0.39, \mathrm{CHCl}_{3}\right) ;{ }^{1} \mathrm{H}$ NMR 7.62 (d, 1, J=7.9), $7.44(\mathrm{~d}, 1$, $J=7.9), 7.41(\mathrm{dd}, 1, J=7.9,7.9), 7.38$ (d, 1, $J=7.9), 7.21(\mathrm{dd}, 1, J=7.9,7.9), 7.21(\mathrm{dd}, 1, J=$ 7.9, 7.9), $7.05(\mathrm{~d}, 1, J=4.9,(\mathrm{CO}) \mathrm{NH}), 6.68(\mathrm{~d}, 1, J=7.9), 6.65$ (dd, 1, J=7.9, 7.9), 5.59 (br s, 2, $\left.\mathrm{NH}_{2}\right), 5.42(\mathrm{~s}, 1), 4.91-4.86(\mathrm{~m}, 1), 4.45(\mathrm{q}, 1, J=6.7), 3.14(\mathrm{dd}, 1, J=12.8,3.1), 1.754(\mathrm{~d}, 3, J=$ 6.7), $1.750(\mathrm{dd}, 1, J=12.8,11.6), 0.90(\mathrm{t}, 9, J=7.9), 0.49$ (q, 6, $J=7.9) ;{ }^{13} \mathrm{C} \mathrm{NMR} 172.8,169.0$, $168.8,148.8,140.0,134.3,132.5,130.7,127.7,125.6,125.0,117.2,116.6,115.5,115.4,83.8$, 79.8, 59.6, 48.9, 41.4, 14.9, 6.9 (3 C), 6.1 (3 C); IR (neat) 3460, 3355, 1738, 1682, 1651; HRMS (Qtof) calcd for $\mathrm{C}_{27} \mathrm{H}_{35} \mathrm{~N}_{4} \mathrm{O}_{4} \mathrm{Si}\left(\mathrm{MH}^{+}\right)$507.2428, found 507.2424.

(2S,4R,5aS,9cS)-4,5,5a,9c-Tetrahydro-2-methyl-4-(4-oxo-3(4H)-quinazolinyl)-5a[(triethylsilyl)oxy]-3H-2a,9b-diazacyclopenta[jk]fluorene-1,3(2H)-dione (TES-

chaetominine, 16). A solution of $15(16.8 \mathrm{mg}, 33 \mu \mathrm{mol})$, triethyl orthoformate $(83 \mu \mathrm{L}$, $500 \mu \mathrm{mol})$ and $\mathrm{TsOH} \cdot \mathrm{H}_{2} \mathrm{O}(1.3 \mathrm{mg}, 7 \mu \mathrm{mol})$ in dry benzene $(3 \mathrm{~mL})$ was refluxed under $\mathrm{N}_{2}$ for 4 h. The solvent was removed under reduced pressure. Flash chromatography on $\mathrm{MeOH}-$ deactivated silica gel (3:1 hexanes/EtOAc) gave $14.2 \mathrm{mg}$ (83\%) of 16 as a white solid: $\mathrm{mp}$ 265$268^{\circ} \mathrm{C} ;[\alpha]^{22}{ }_{\mathrm{D}}-40.0\left(c 0.24, \mathrm{CHCl}_{3}\right) ;{ }^{1} \mathrm{H} \operatorname{NMR}\left(21.5^{\circ} \mathrm{C}\right) 8.30(\mathrm{~d}, 1, J=7.9), 7.85(\mathrm{~s}, 1), 7.77$ 
(dd, 1, $J=7.9,7.9), 7.71(\mathrm{~d}, 1, J=7.9), 7.66(\mathrm{~d}, 1, J=7.9), 7.51(\mathrm{dd}, 1, J=7.9,7.9), 7.45$ (dd, 1, $J=7.9,7.9), 7.35(\mathrm{~d}, 1, J=7.9), 7.23(\mathrm{dd}, 1, J=7.9,7.9), 5.48(\mathrm{~s}, 1), 4.46(\mathrm{q}, 1, J=7.3), 3.05-$ 2.30 (br, 1), 2.65 (br d, 1, $J=12.0), 1.76$ (d, 3, $J=7.3), 0.90(\mathrm{t}, 9, J=7.9), 0.57-0.41(\mathrm{~m}, 6)\left(\mathrm{H}_{14}\right.$ was not observed due to slow rotation about the $\mathrm{C}_{14}-\mathrm{N}$ bond); $\left(30^{\circ} \mathrm{C}\right) 8.30(\mathrm{~d}, 1, J=7.9), 7.85(\mathrm{~s}$, 1), $7.76(\mathrm{dd}, 1, J=7.9,7.9), 7.71(\mathrm{~d}, 1, J=7.9), 7.66$ (d, 1, $J=7.9), 7.50$ (dd, 1, J=7.9, 7.9), $7.44(\mathrm{dd}, 1, J=7.9,7.9), 7.35(\mathrm{~d}, 1, J=7.9), 7.22(\mathrm{dd}, 1, J=7.9,7.9), 5.48(\mathrm{~s}, 1), 4.45(\mathrm{q}, 1, J=$ 7.3), 2.94-2.50 (br, 1), 2.64 (br d, 1, $J=12.0$ ), 1.76 (d, 3, $J=7.3$ ), 0.90 (t, 9, $J=7.9$ ), 0.57-0.42 $(\mathrm{m}, 6)\left(\mathrm{H}_{14}\right.$ was not observed due to slow rotation about the $\mathrm{C}_{14}-\mathrm{N}$ bond); ${ }^{13} \mathrm{C}$ NMR $172.3,160.8$, 147.6, 147.3 (br), 140.0, 134.5, 133.8, 131.1, 127.5, 127.4, 127.0, 125.7, 124.8, 122.0 (br), 115.7, 83.7, 79.8, 59.9, 50.0 (br), 41.1 (br), 14.6, 6.8 (3 C), 6.0 (3 C) (one amide carbonyl carbon was not observed due to slow rotation about the $\mathrm{C}_{14}-\mathrm{N}$ bond); IR (neat) $1732,1688,1682,1613$; HRMS (Qtof) calcd for $\mathrm{C}_{28} \mathrm{H}_{33} \mathrm{~N}_{4} \mathrm{O}_{4} \mathrm{Si}\left(\mathrm{MH}^{+}\right)$517.2271, found 517.2256.

$(2 S, 4 R, 5 a S, 9 c S)-4,5,5 a, 9 c-T e t r a h y d r o-5 a-h y d r o x y-2-m e t h y l-4-(4-0 x o-3(4 H)-$ quinazolinyl)-3H-2a,9b-diazacyclopenta[jk]fluorene-1,3(2H)-dione (chaetominine, 1 ). TESChaetominine (16) $(13.8 \mathrm{mg}, 27 \mu \mathrm{mol})$ was treated with $0.8 \mathrm{~mL}$ of a 1:19 mixture of hydrofluoric acid (49\% wt. in $\left.\mathrm{H}_{2} \mathrm{O}\right)$ and acetonitrile at $25^{\circ} \mathrm{C}$. The mixture was stirred for $7 \mathrm{~h}$ at $25^{\circ} \mathrm{C}$ and diluted with EtOAc $(10 \mathrm{~mL})$. The organic layer was washed with saturated $\mathrm{NaHCO}_{3}$ solution and brine, dried $\left(\mathrm{MgSO}_{4}\right)$, and concentrated. Flash chromatography on $\mathrm{MeOH}-$ deactivated silica gel (1:1 hexanes/EtOAc) gave $9.6 \mathrm{mg}(89 \%)$ of 1 as a white solid: $\mathrm{mp}$ 162$165^{\circ} \mathrm{C}$; $\left\{\right.$ lit. $\left.{ }^{1} \mathrm{mp} 161-163{ }^{\circ} \mathrm{C}\right\} ;[\alpha]^{22}{ }_{\mathrm{D}}-49.4$ (c 0.26, MeOH); $\left\{\right.$ lit. ${ }^{1}[\alpha]^{20}{ }_{\mathrm{D}}-70(c$ 0.48, MeOH$\left.)\right\}$; $\mathrm{CD}($ c $0.0051 \mathrm{mg} / \mathrm{mL}$ in $\mathrm{MeOH}) \lambda 203(\Delta \varepsilon+16.7), 214(\Delta \varepsilon-12.0), 228(\Delta \varepsilon+4.1), 234(\Delta \varepsilon+4.7)$, $241(\Delta \varepsilon+2.3), 251(\Delta \varepsilon+4.3), 285(\Delta \varepsilon-3.3) ;{ }^{1} \mathrm{H} \operatorname{NMR}\left(21.5^{\circ} \mathrm{C}\right) 8.25(\mathrm{~d}, 1, J=7.9), 7.88(\mathrm{~s}, 1)$, $7.77(\mathrm{dd}, 1, J=7.9,7.9), 7.70(\mathrm{~d}, 1, J=7.9), 7.65(\mathrm{~d}, 1, J=7.9), 7.50$ (dd, 1, $J=7.9,7.9), 7.44$ $(\mathrm{dd}, 1, J=7.9,7.9), 7.40(\mathrm{~d}, 1, J=7.9), 7.24(\mathrm{dd}, 1, J=7.9,7.9), 5.54(\mathrm{~s}, 1), 4.47(\mathrm{q}, 1, J=6.7)$, 3.63-3.41 (br, 1, OH), 3.01-2.67 (br, 1), 2.66 (br d, 1, $J=11.6), 1.77$ (d, 3, J=6.7) (H $\mathrm{H}_{14}$ was not observed due to slow rotation about the $\mathrm{C}_{14}-\mathrm{N}$ bond); ${ }^{1} \mathrm{H}$ NMR (DMSO- $\left.d_{6}, 21.5{ }^{\circ} \mathrm{C}\right) 8.28$ (br s, 1), 8.17 (br d, 1, $J=7.9$ ), 7.86 (dd, 1, $J=7.9,7.9$ ), 7.69 (d, 1, $J=7.9), 7.58$ (dd, 1, $J=7.9,7.9$ ), 
$7.51(\mathrm{~d}, 1, J=7.9), 7.49(\mathrm{~d}, 1, J=7.9), 7.43(\mathrm{dd}, 1, J=7.9,7.9), 7.25(\mathrm{dd}, 1, J=7.9,7.9), 6.70$ (s, 1, OH), 6.02-5.78 (br, 1), $5.60(\mathrm{~s}, 1), 4.61$ (q, 1, J=6.7), 2.93 (dd, 1, J=12.8, 12.8), 2.53 (d, $1, J=12.8), 1.59(\mathrm{~d}, 3, J=6.7)$; $\left(\mathrm{DMSO}_{6}, 30^{\circ} \mathrm{C}\right) 8.28($ br s, 1$), 8.18$ (br d, $\left.1, J=7.9\right), 7.86$ $(\mathrm{dd}, 1, J=7.9,7.9), 7.69(\mathrm{~d}, 1, J=7.9), 7.58(\mathrm{dd}, 1, J=7.9,7.9), 7.51(\mathrm{~d}, 1, J=7.9), 7.49$ (d, 1, $J$ = 7.9), $7.43(\mathrm{dd}, 1, J=7.9,7.9), 7.25(\mathrm{dd}, 1, J=7.9,7.9), 6.69$ (s, 1, OH), 6.03-5.76 (br, 1), 5.60 $(\mathrm{s}, 1), 4.61(\mathrm{q}, 1, J=6.7), 2.93(\mathrm{dd}, 1, J=12.8,12.8), 2.53(\mathrm{dd}, 1, J=12.8,3.1), 1.60(\mathrm{~d}, 3, J=$ 6.7); ${ }^{13} \mathrm{C}$ NMR (DMSO- $\left.d_{6}, 500 \mathrm{MHz}, 30{ }^{\circ} \mathrm{C}\right) 172.0,165.4,160.0,147.4,146.7,138.7,136.7$, $134.6,129.8,127.20,127.17,126.3,125.4,124.8,121.1,114.4,82.5,76.3,59.5,49.9,38.1,13.9$ (Our ${ }^{13} \mathrm{C}$ NMR data are identical to those of the natural product ${ }^{1}$ except that they are 0.5 to 0.6 ppm upfield indicating that they are referenced differently); IR (neat) 3354, 1732, 1690, 1681, 1674, 1668, 1610; HRMS (Qtof) calcd for $\mathrm{C}_{22} \mathrm{H}_{19} \mathrm{~N}_{4} \mathrm{O}_{4}\left(\mathrm{MH}^{+}\right)$403.1406, found 403.1487.

Methyl ( $\alpha S, 3 R, 4 a S, 9 a S)-2,3,4,4 a, 9,9 a-H e x a h y d r o-4 a-h y d r o x y-\alpha-m e t h y l-2-o x o-3-(4-$ oxo-3(4H)-quinazolinyl)-1H-pyrido[2,3-b]indole-1-ethanoate (17). A solution of 1 in $\mathrm{MeOH}$ was heated at reflux for $5 \mathrm{~h}$. The solvent was removed under reduced pressure to yield a 1:1 mixture of chaetominine (1) and the corresponding amino ester 17. Flash chromatography on $\mathrm{MeOH}$-deactivated silica gel (1:1 hexanes/EtOAc) gave a 6:1 mixture of $\mathbf{1 7}$ and $\mathbf{1}$, preceded by fractions rich in chaetominine (1). Heating the 6:1 mixture of $\mathbf{1 7}$ and $\mathbf{1}$ in $\mathrm{MeOH}$ at reflux for 5 h regenerated a 1:1 mixture of $\mathbf{1 7}$ and $\mathbf{1}$.

Data for 17: ${ }^{1} \mathrm{H}$ NMR $8.23(\mathrm{~d}, 1, J=7.6), 7.91(\mathrm{~s}, 1), 7.74(\mathrm{dd}, 1, J=7.6,7.6), 7.68(\mathrm{~d}, 1$, $J=7.6), 7.47(\mathrm{dd}, 1, J=7.6,7.6), 7.23(\mathrm{~d}, 1, J=7.6), 7.16(\mathrm{dd}, 1, J=7.6,7.6), 6.85(\mathrm{dd}, 1, J=$ 7.6, 7.6), 6.74 (d, 1, J=7.6), 5.48-5.29 (br, 1, NH), 5.11 (s, 1), 4.92-4.80 (br, 1), 4.68-4.61 (br, 1), 3.77 (s, 3), 3.17 (br s, 1, OH), 2.98-2.83 (br, 1), 2.36 (dd, 1, J=13.1, 5.2), 1.62 (d, 3, J= 7.3).

\section{References and Notes}

10. Hannick, S. M.; Kishi, Y. J. Org. Chem. 1983, 48, 3833-3835. 
Synthesis of Chaetominine

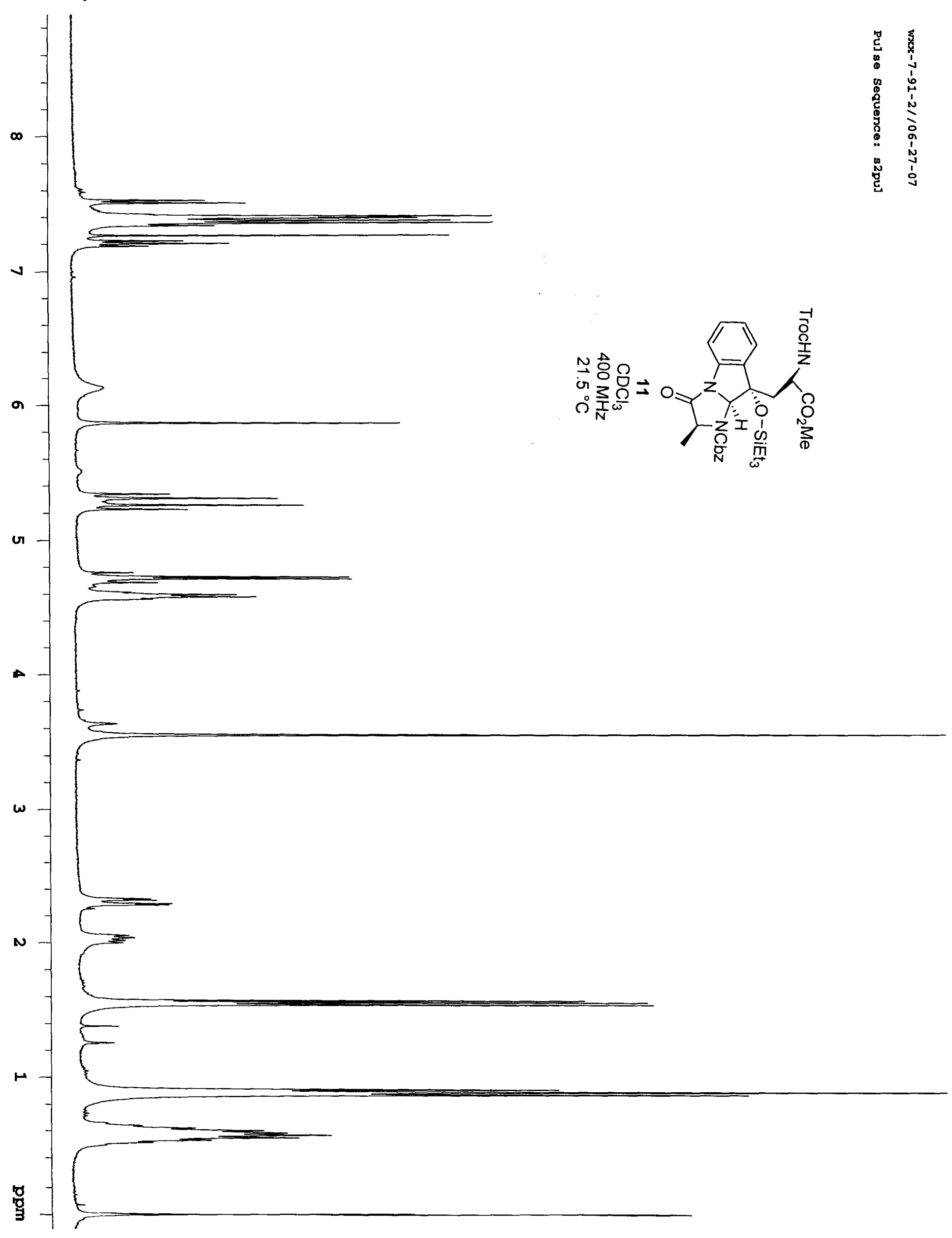




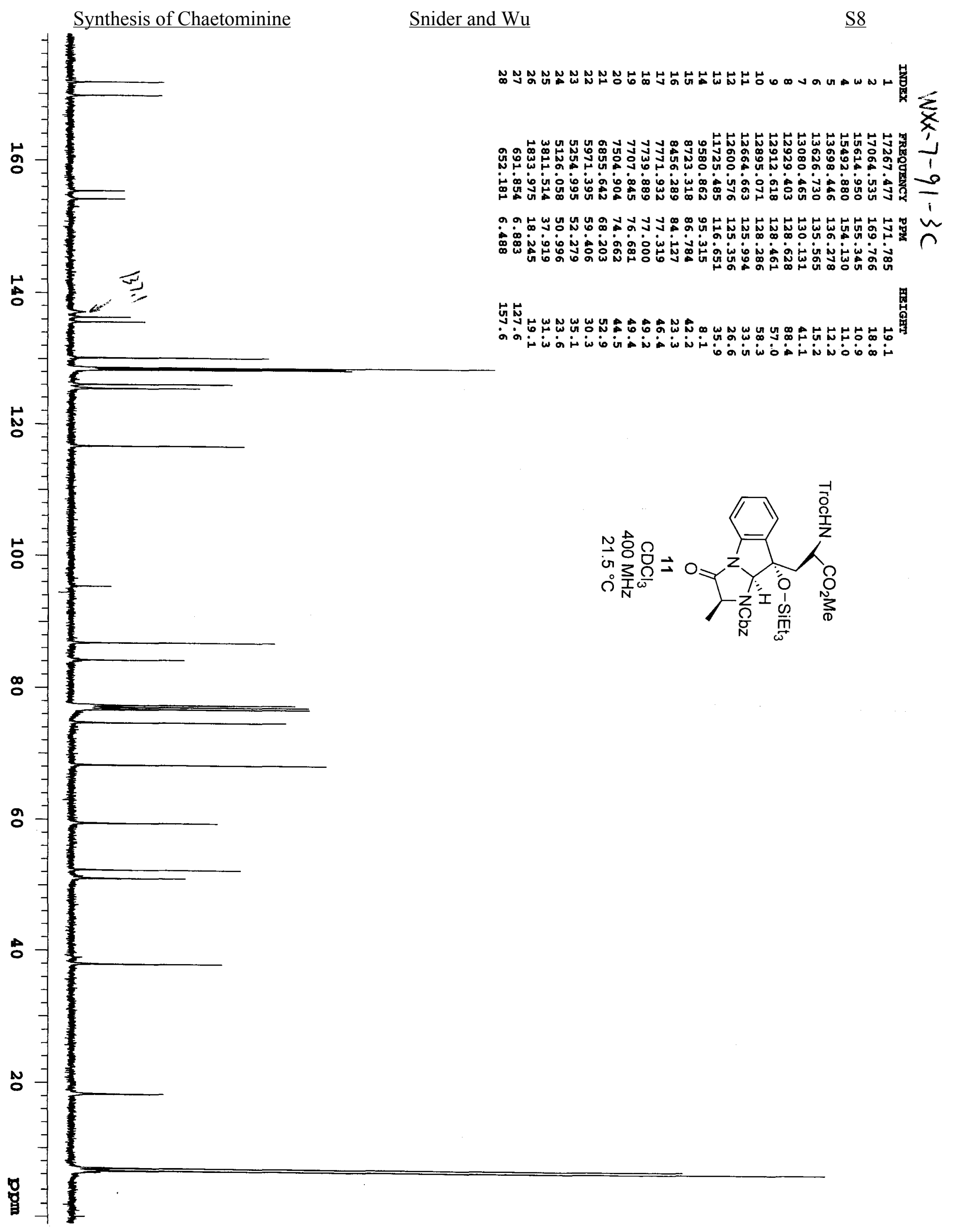




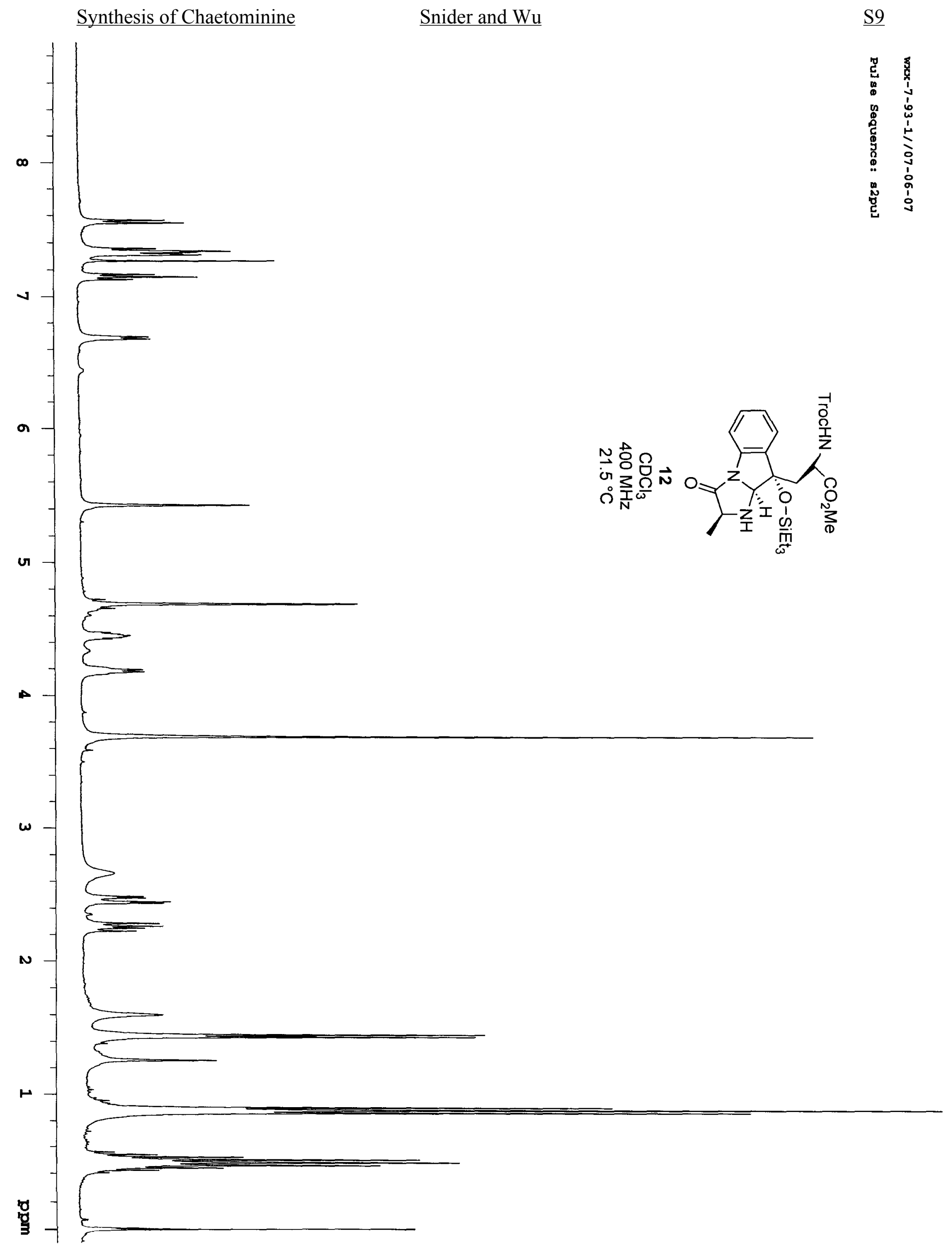




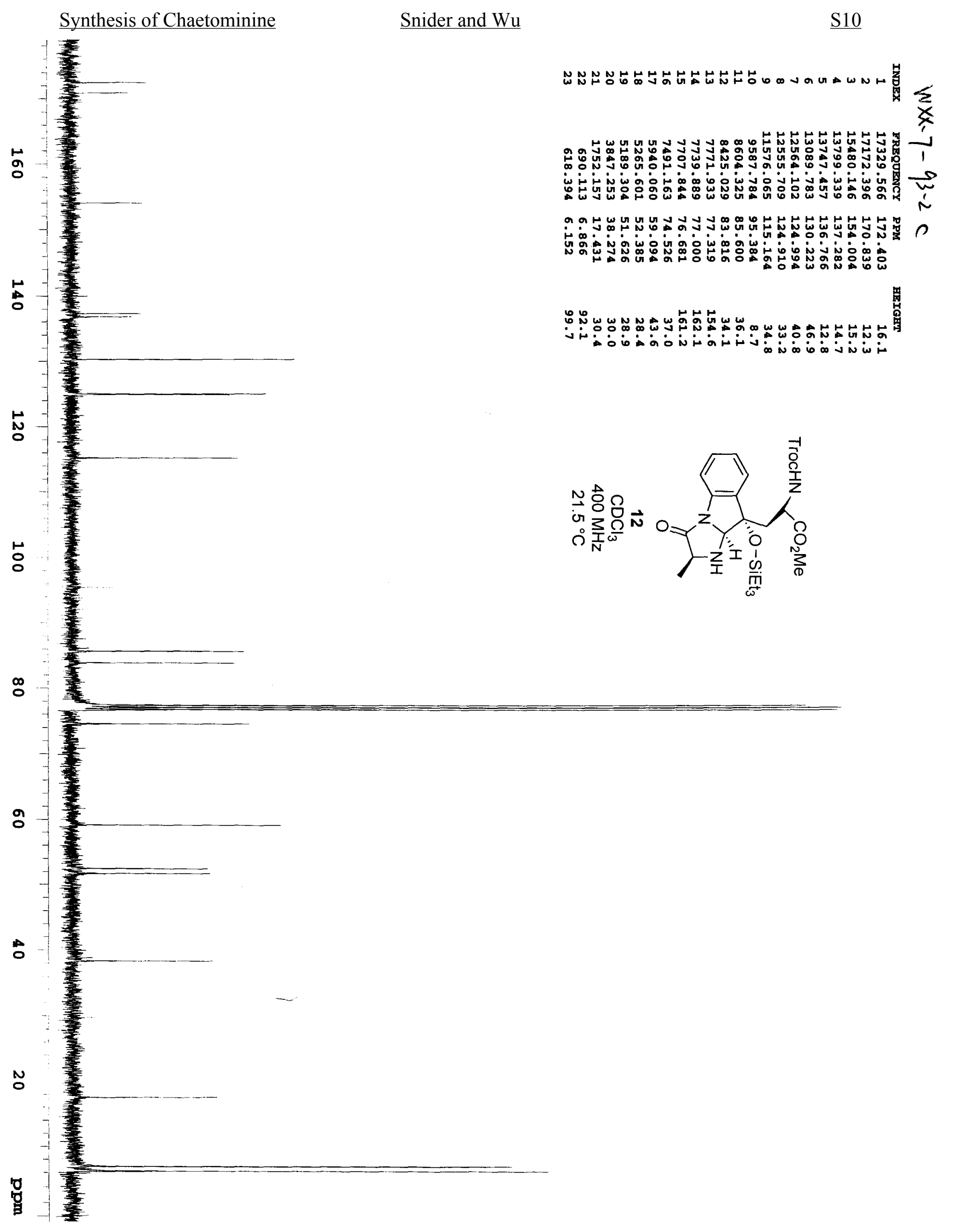


Synthesis of Chaetominine

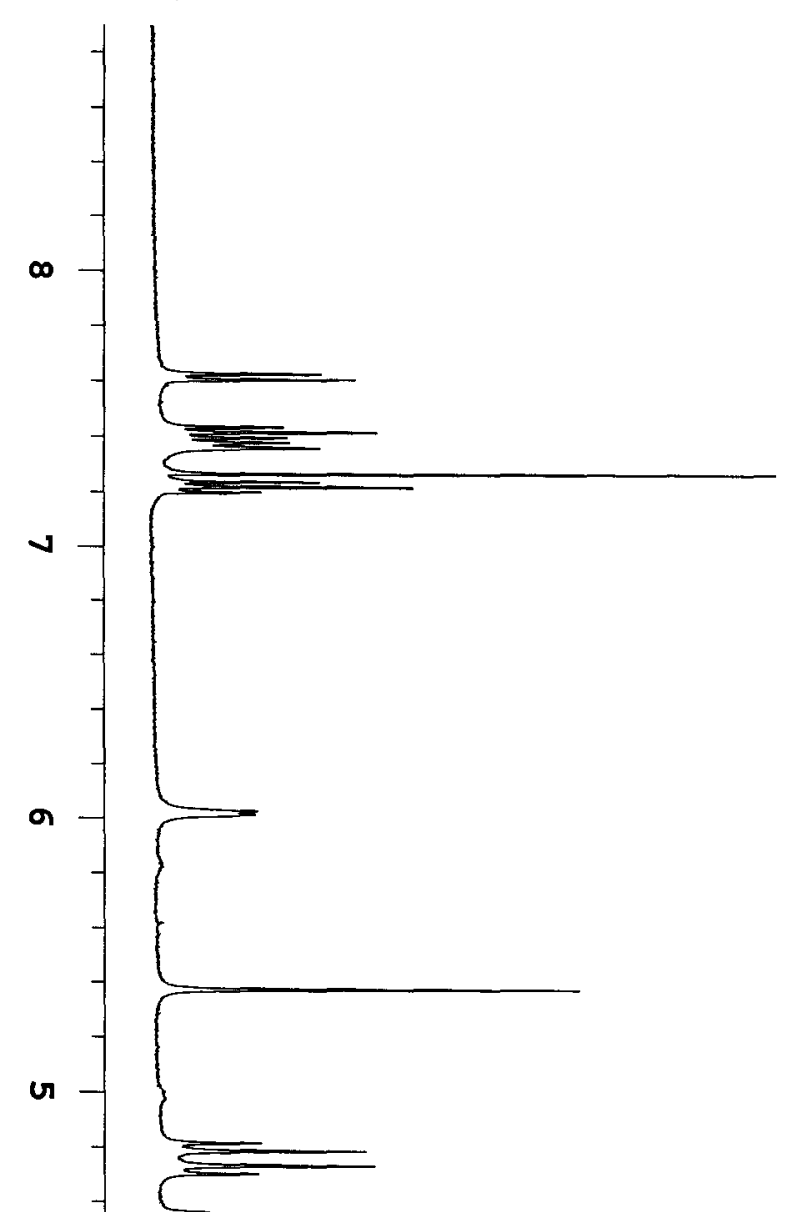

$\underline{\text { Snider and Wu }}$

$\underline{\mathrm{S} 11}$

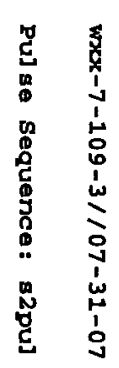

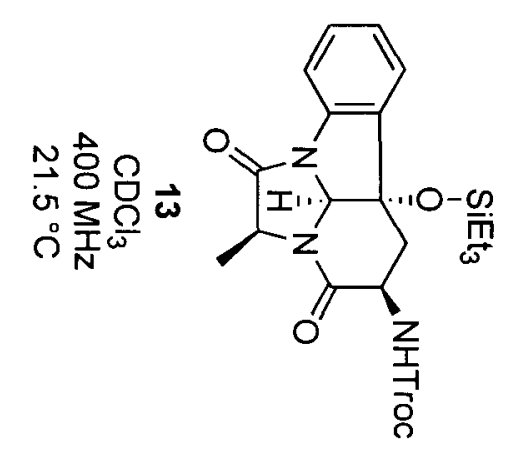

w 


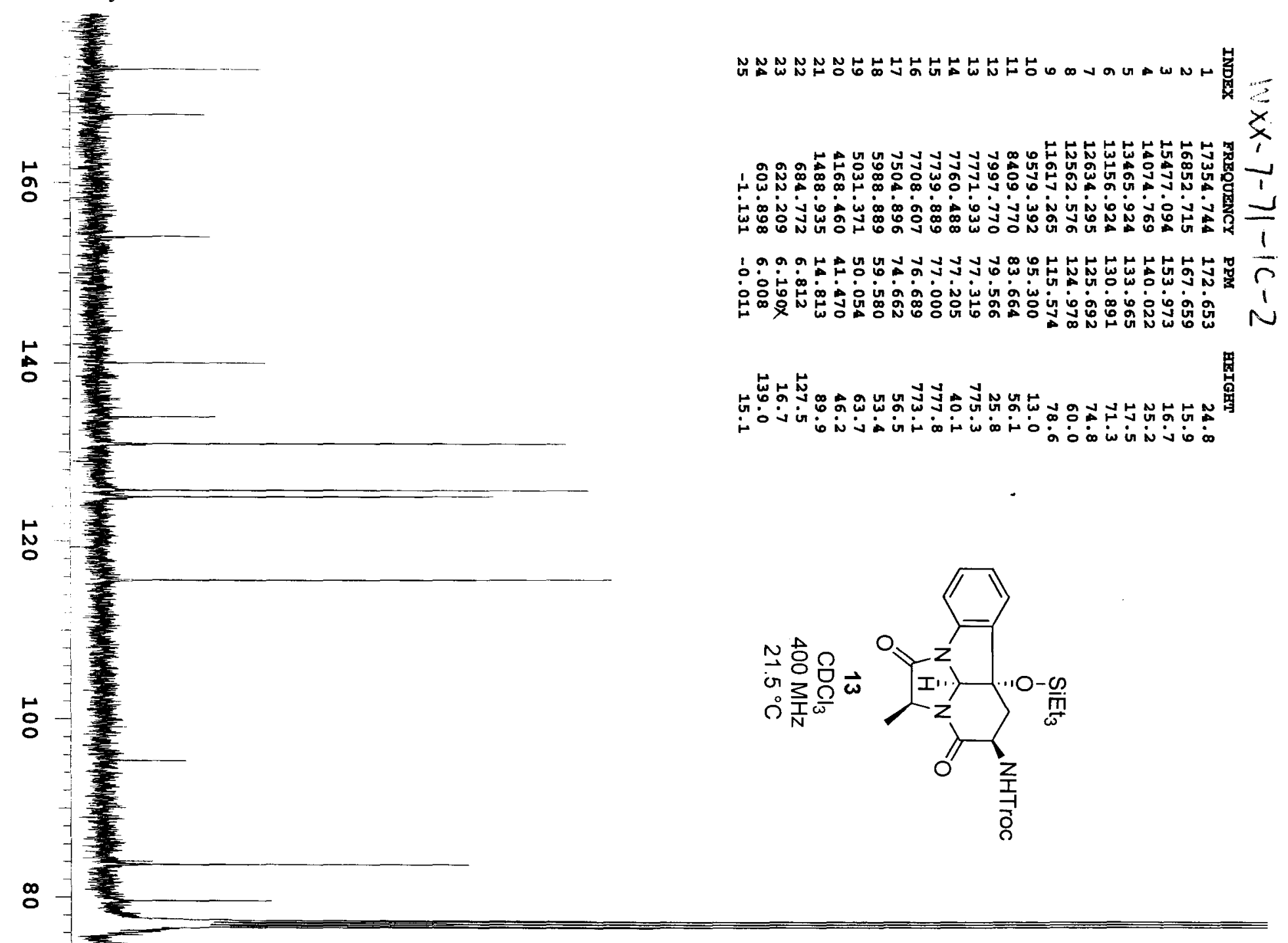


Synthesis of Chaetominine

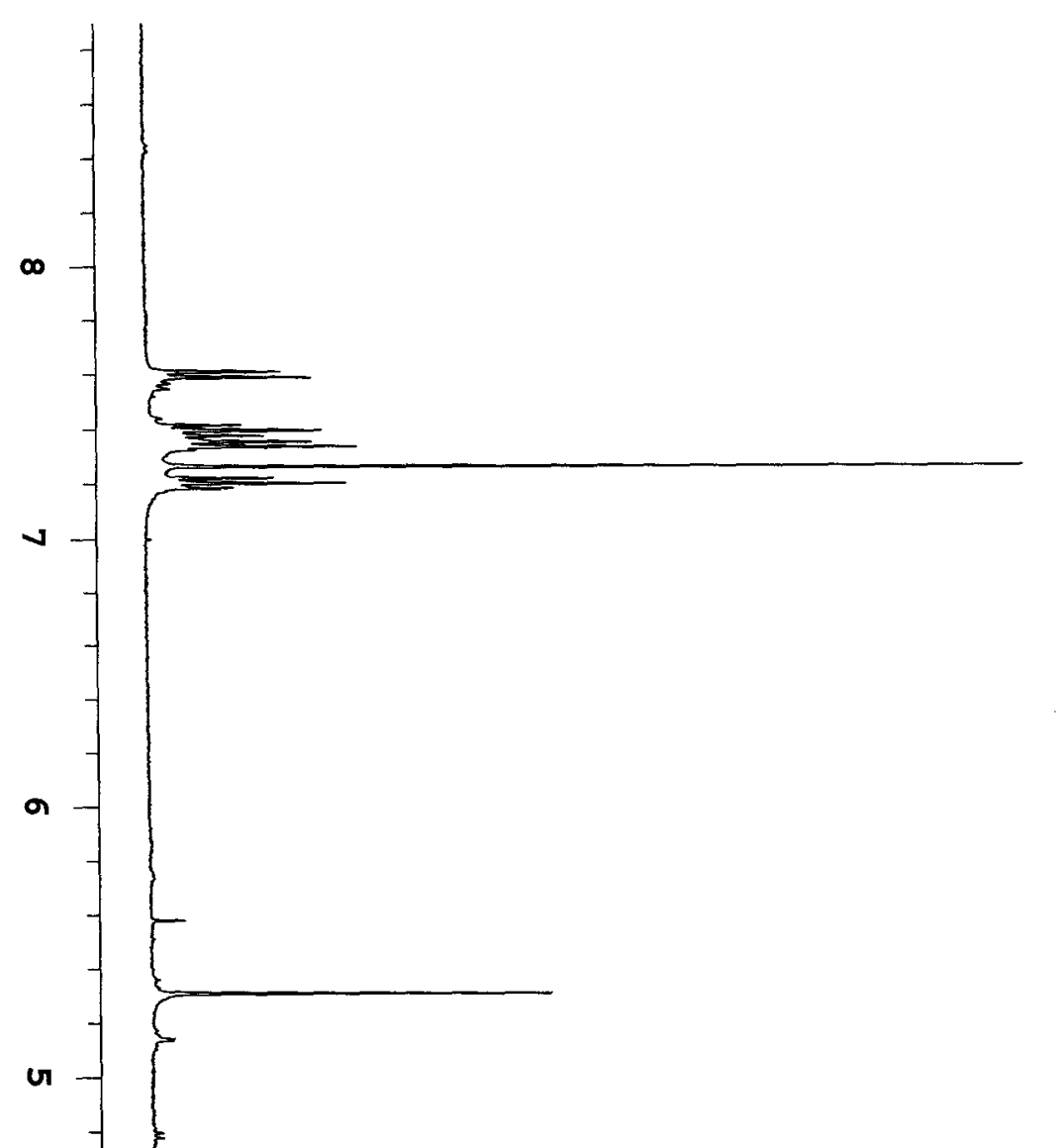

$\underline{\text { Snider and Wu }}$

$\underline{\mathrm{S} 13}$

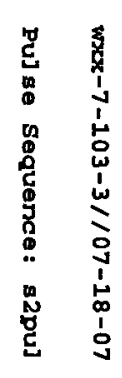

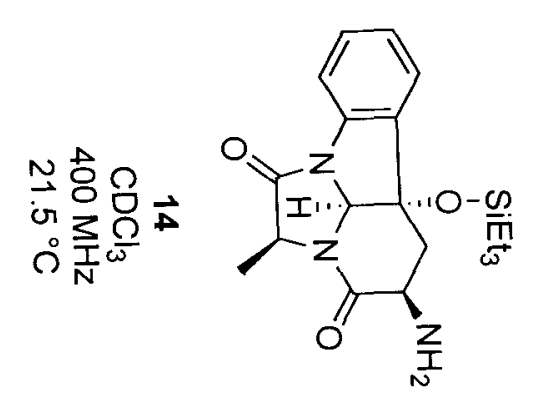


Synthesis of Chaetominine

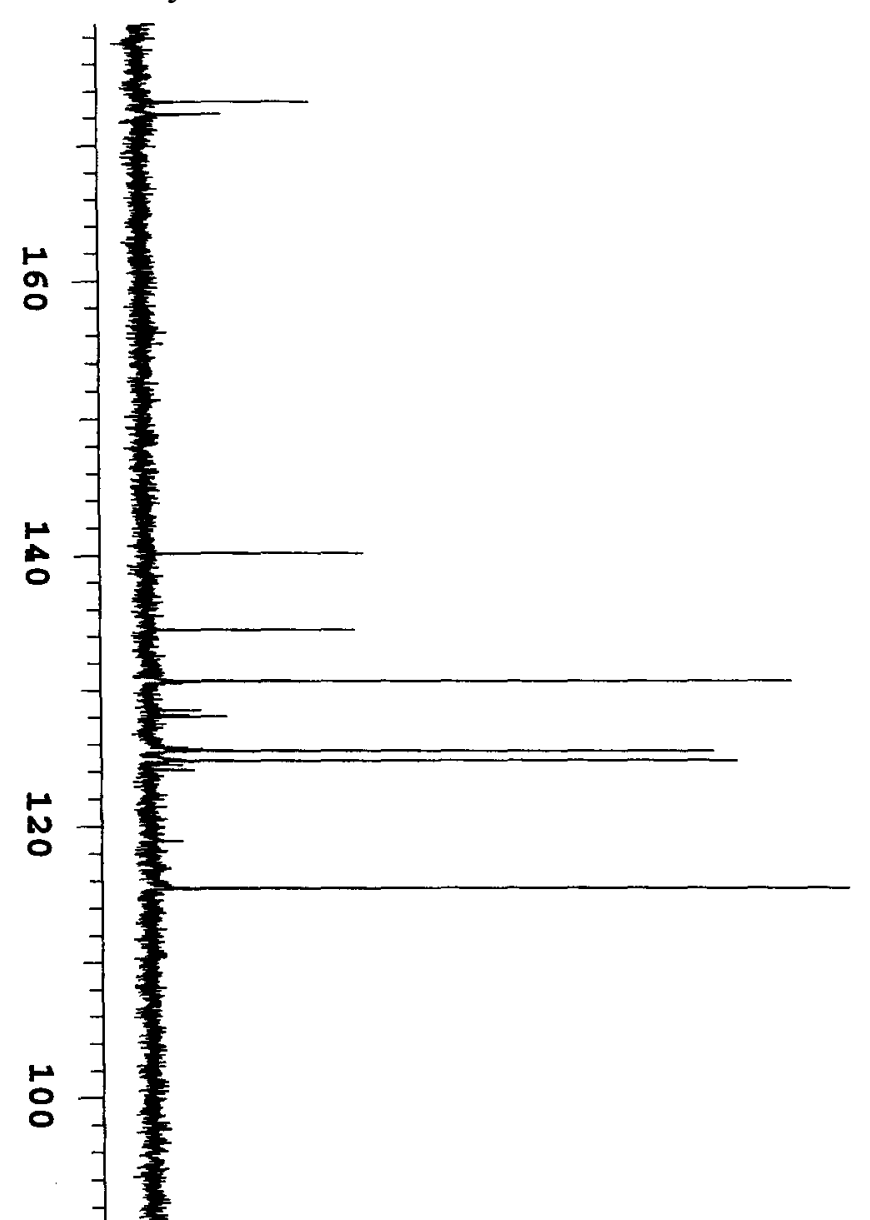

$\underline{\text { Snider and } \mathrm{Wu}}$

$\underline{\mathrm{S} 14}$

N N 最

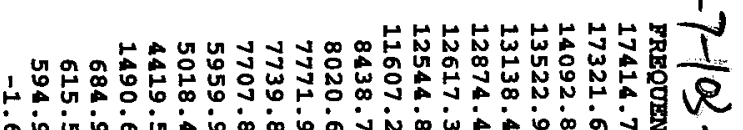

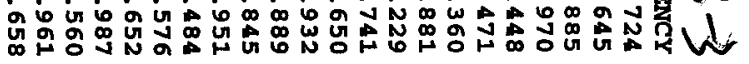
药

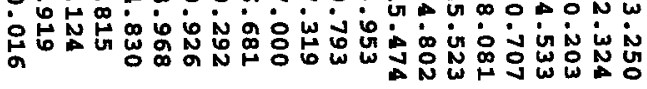

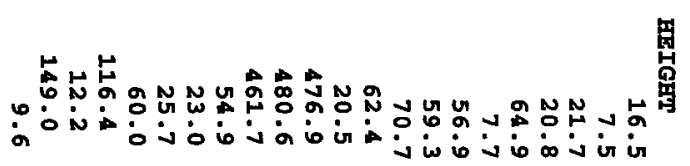

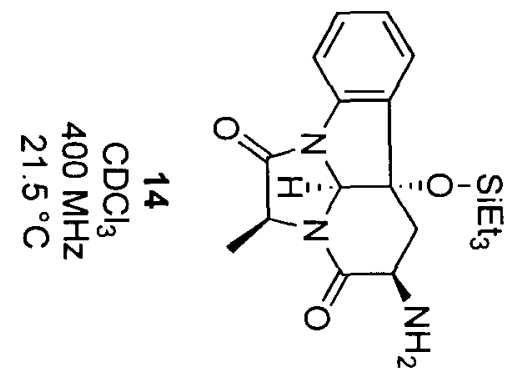


Synthesis of Chaetominine

$\infty$

$\checkmark$

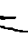

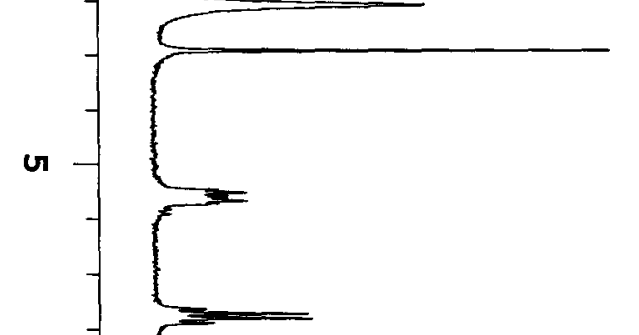

i

$\omega$ $\underline{\text { Snider and } \mathrm{Wu}}$

$\underline{\mathrm{S} 15}$

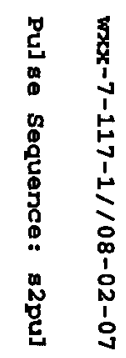

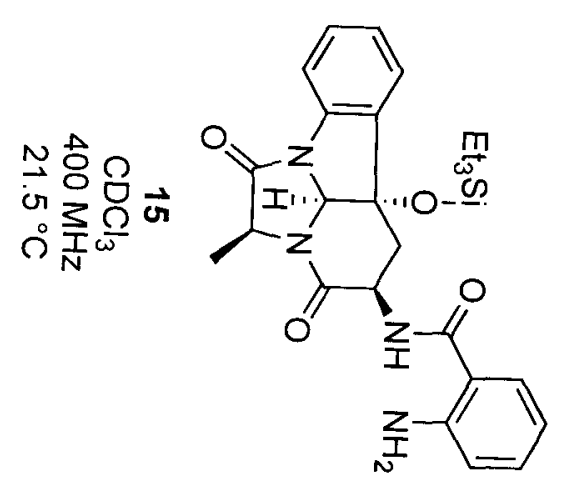


Synthesis of Chaetominine

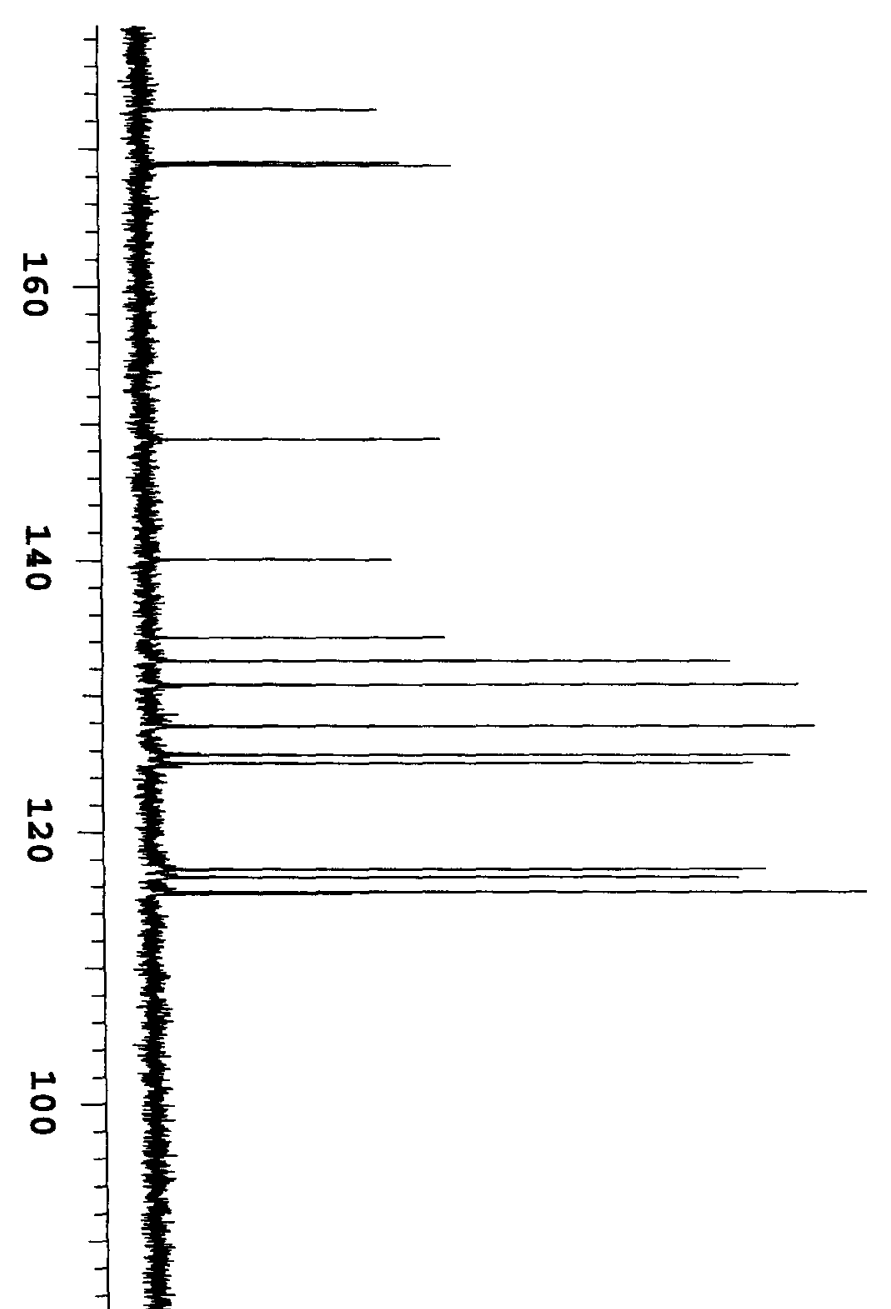

$\underline{\text { Snider and } \mathrm{Wu}}$

$\underline{\mathrm{S} 16}$

N

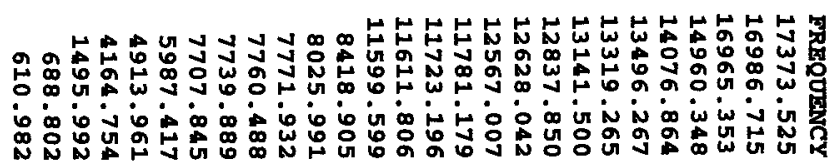

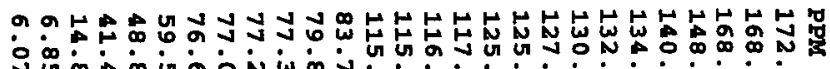

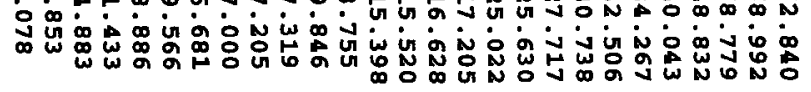

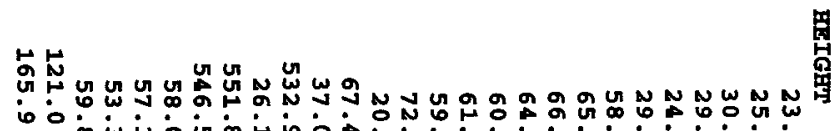

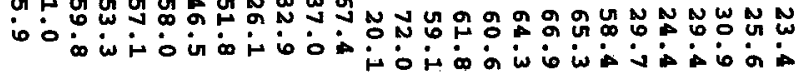

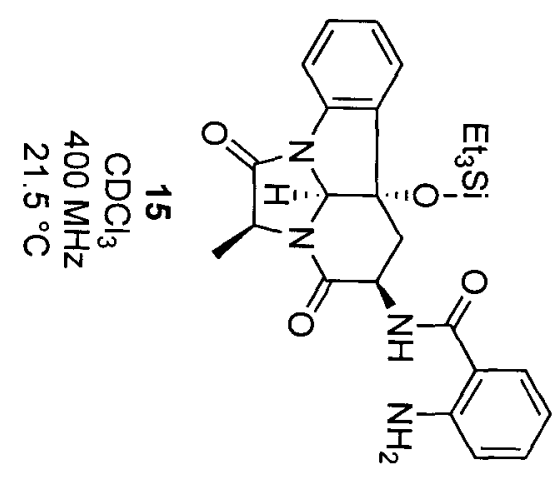

:

읭

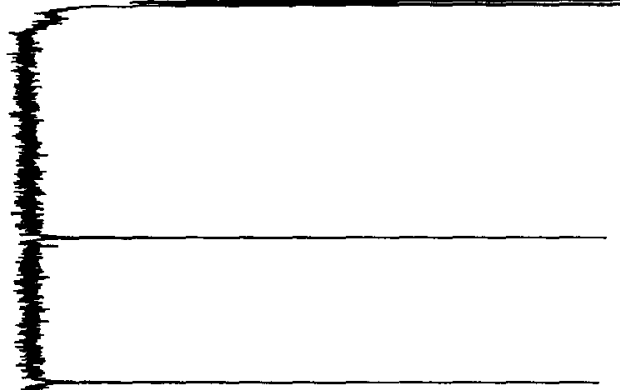

吕

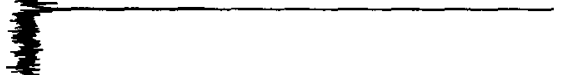

今

g- 
Synthesis of Chaetominine

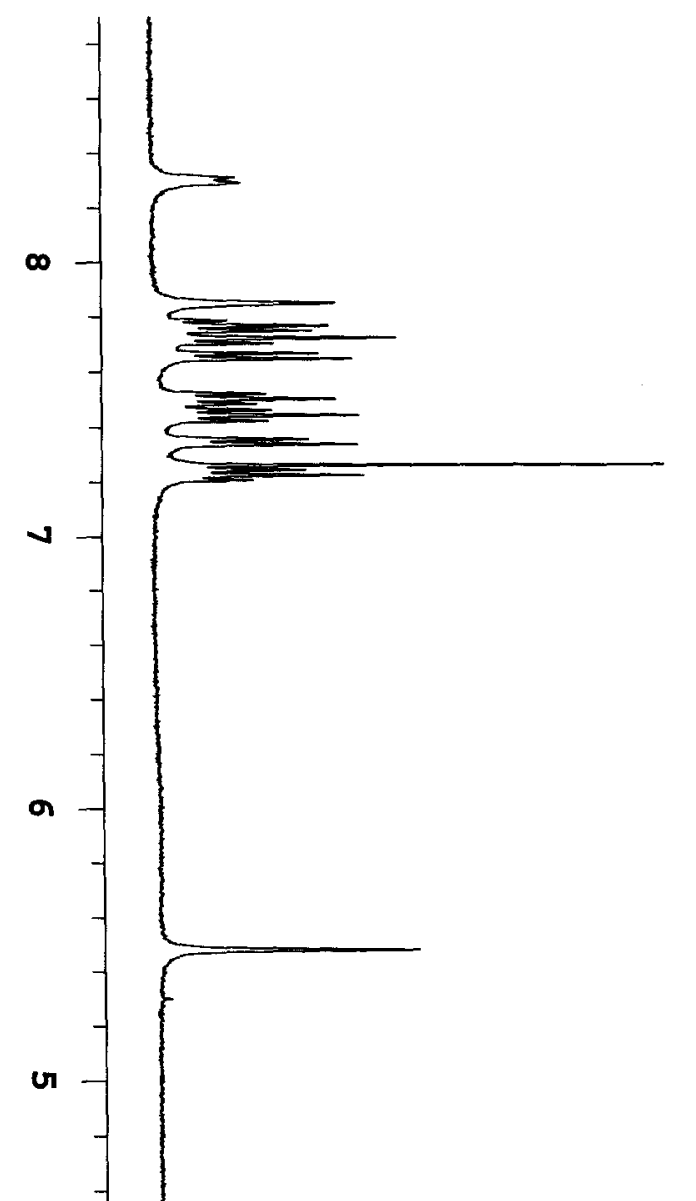

$\underline{\text { Snider and } \mathrm{Wu}}$ 
Synthesis of Chaetominine

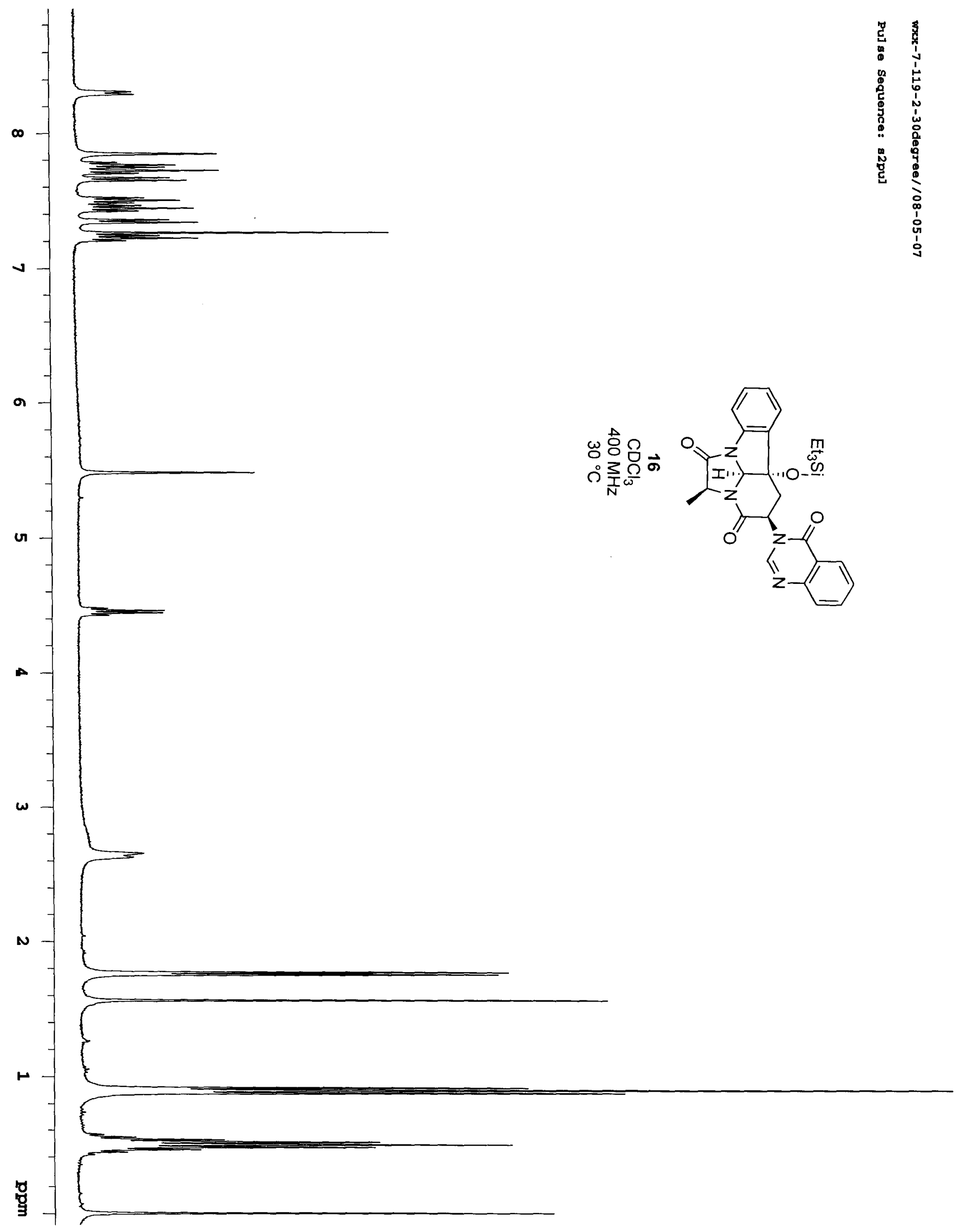




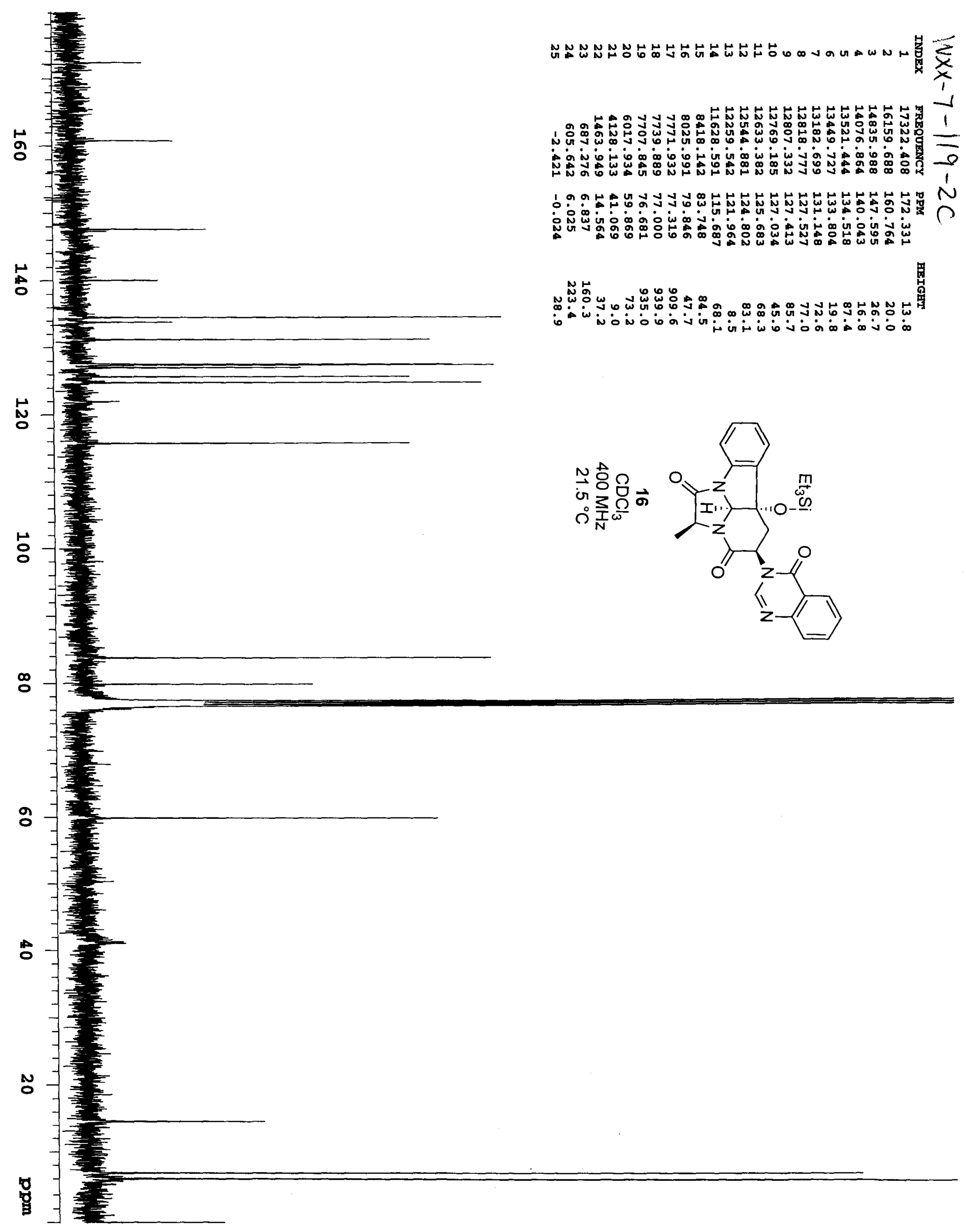


Synthesis of Chaetominine

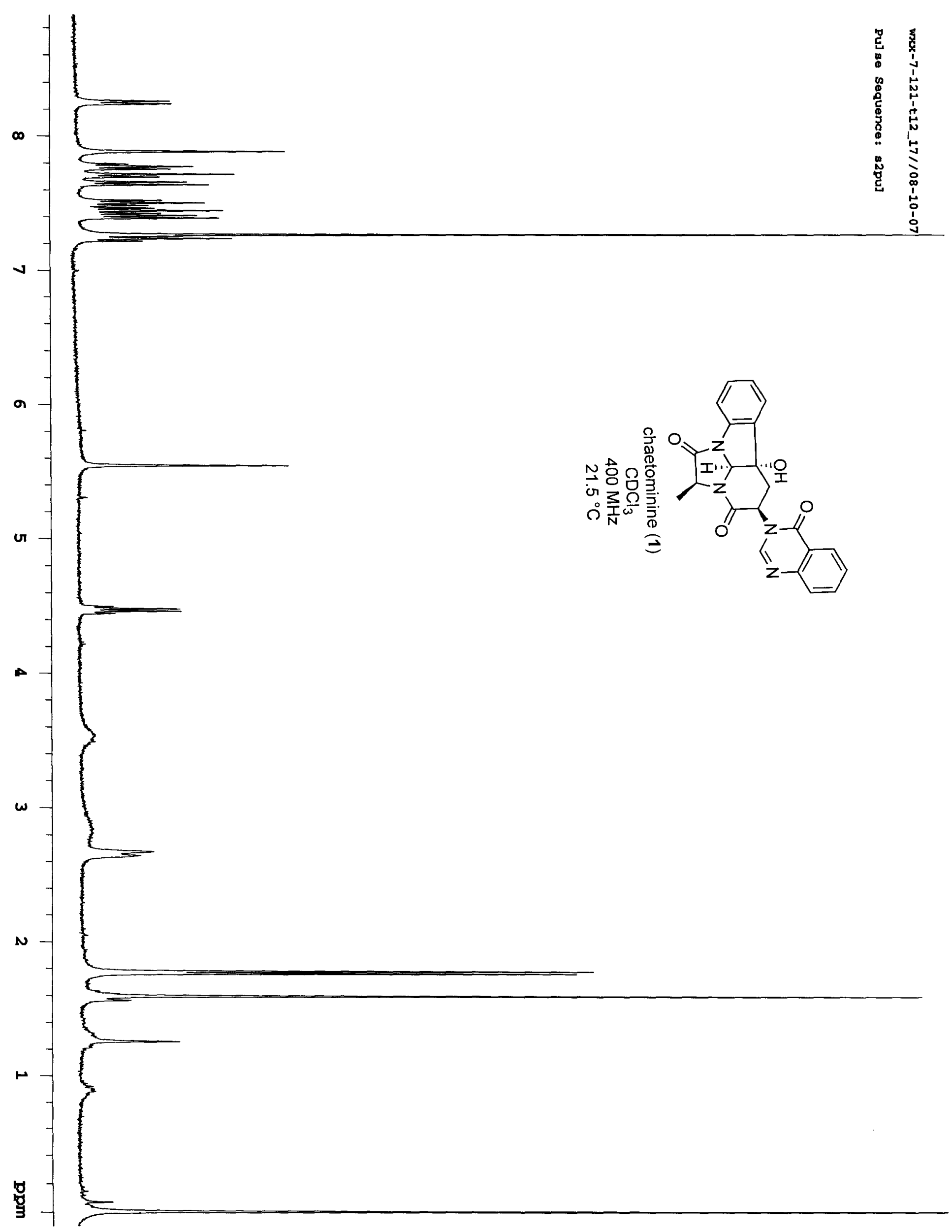


Synthesis of Chaetominine

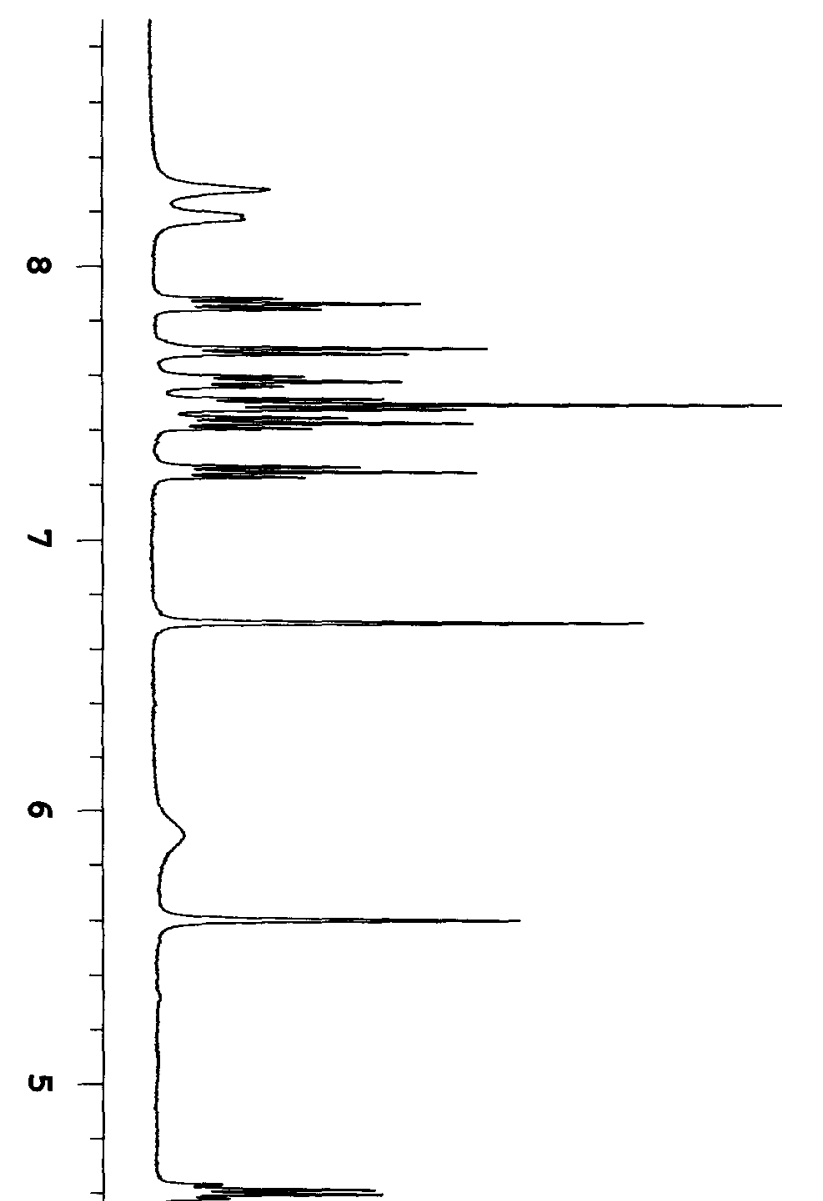

$\underline{\text { Snider and } \mathrm{Wu}}$

$\underline{\mathrm{S} 21}$

$\begin{array}{ll}0 & 1 \\ 0 & 0 \\ 0 & 1 \\ 0 & 1 \\ 0 & 0 \\ 0 & 0 \\ 0 & 0 \\ 0 & \omega \\ 0 & \vdots \\ 0 & 0 \\ 0 & 0 \\ 0 & 0 \\ 0 & 0 \\ 0 & 1 \\ 0 & 0\end{array}$

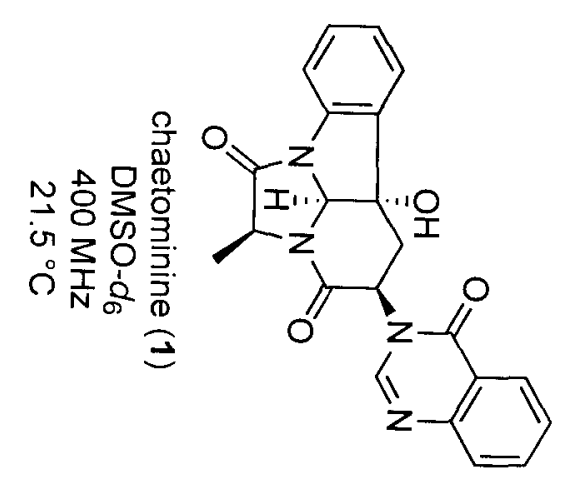




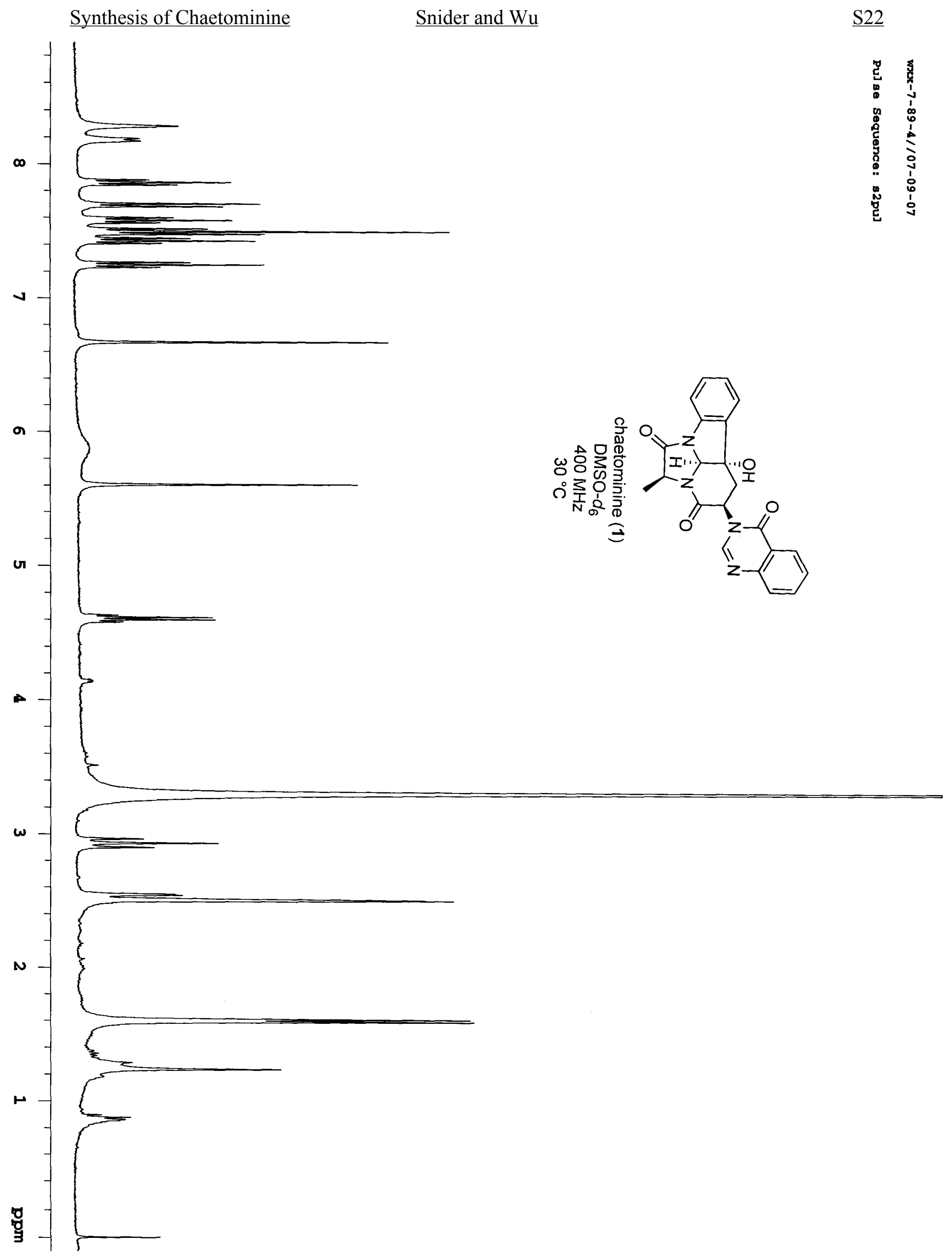




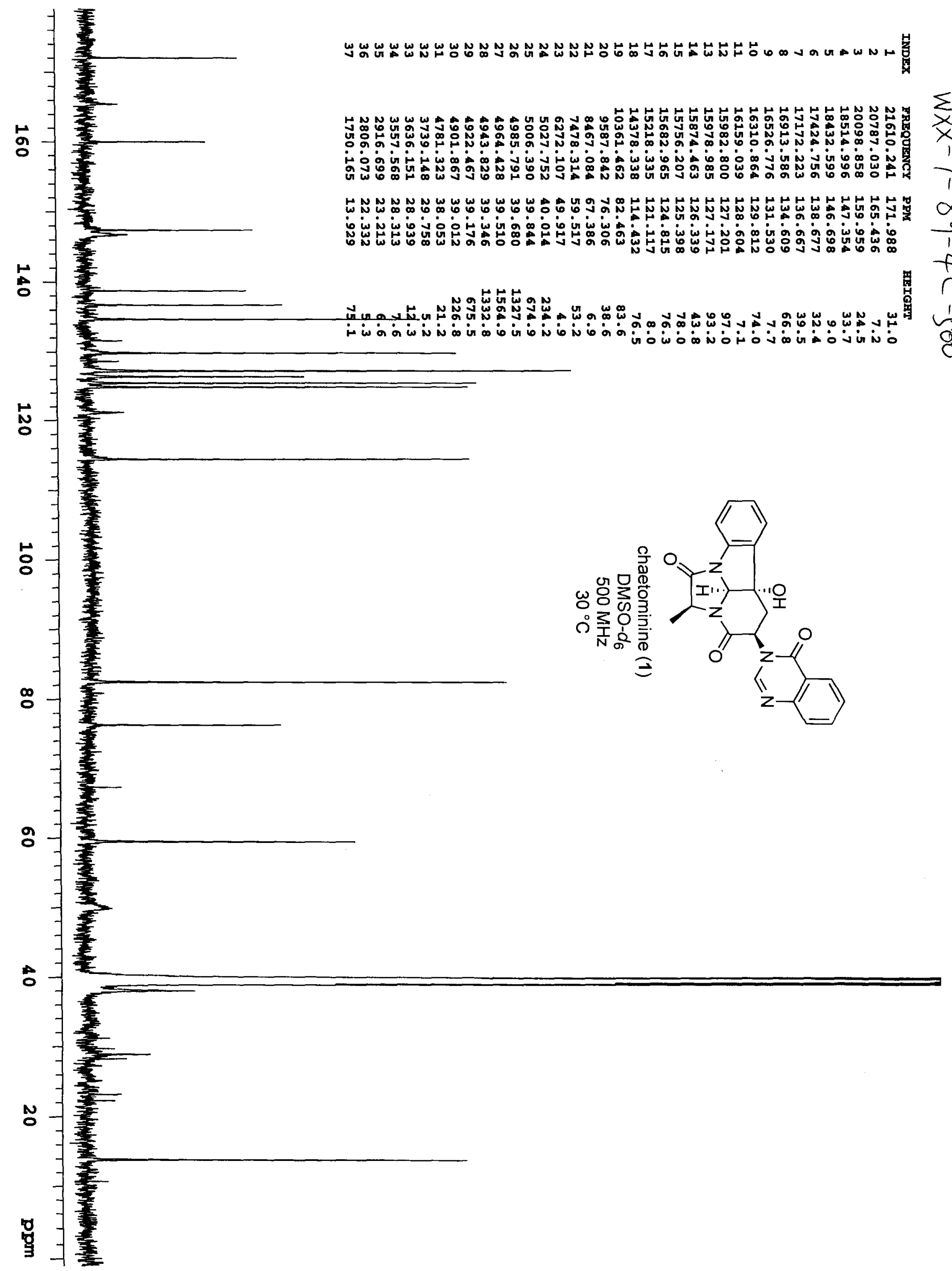




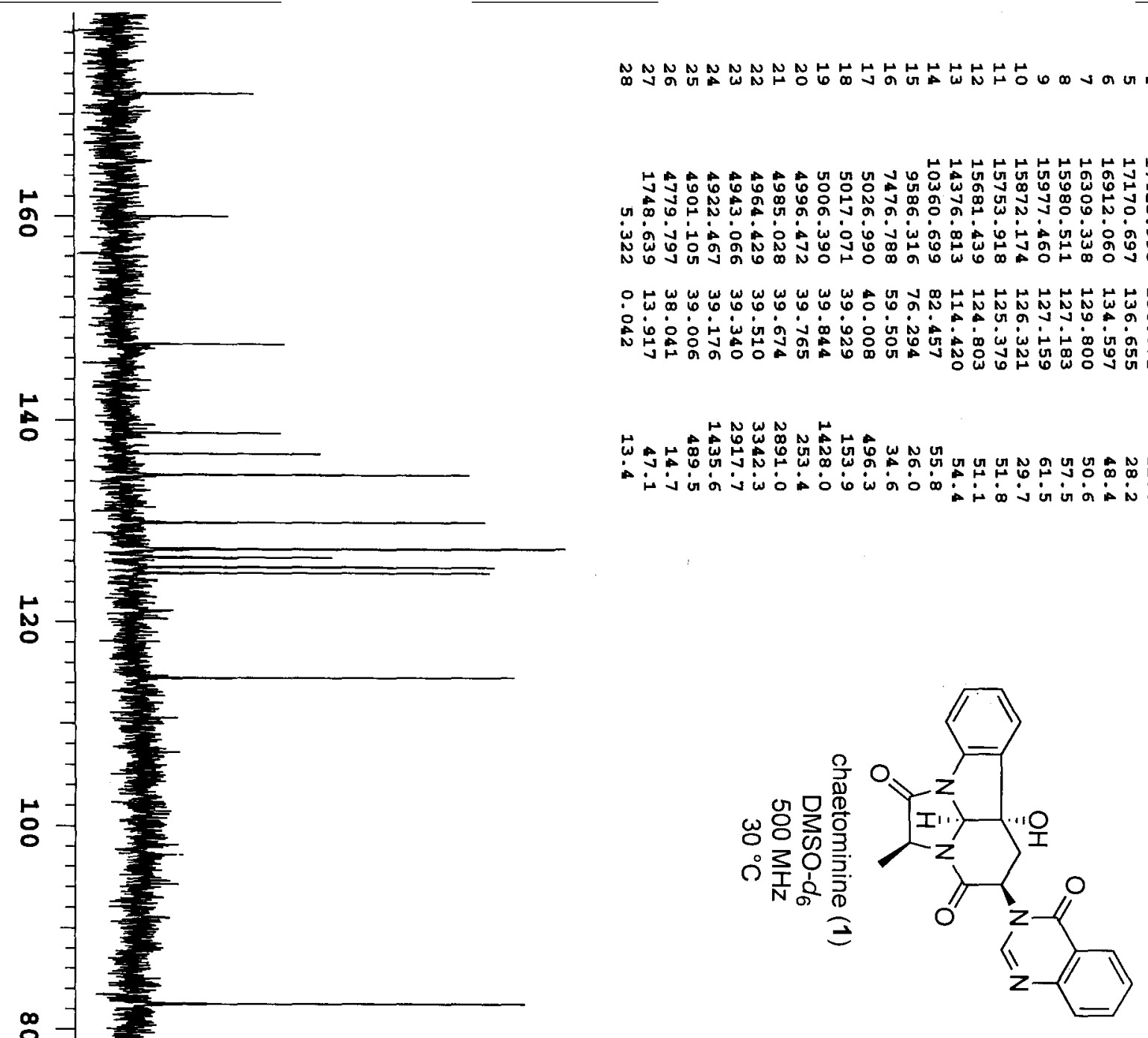




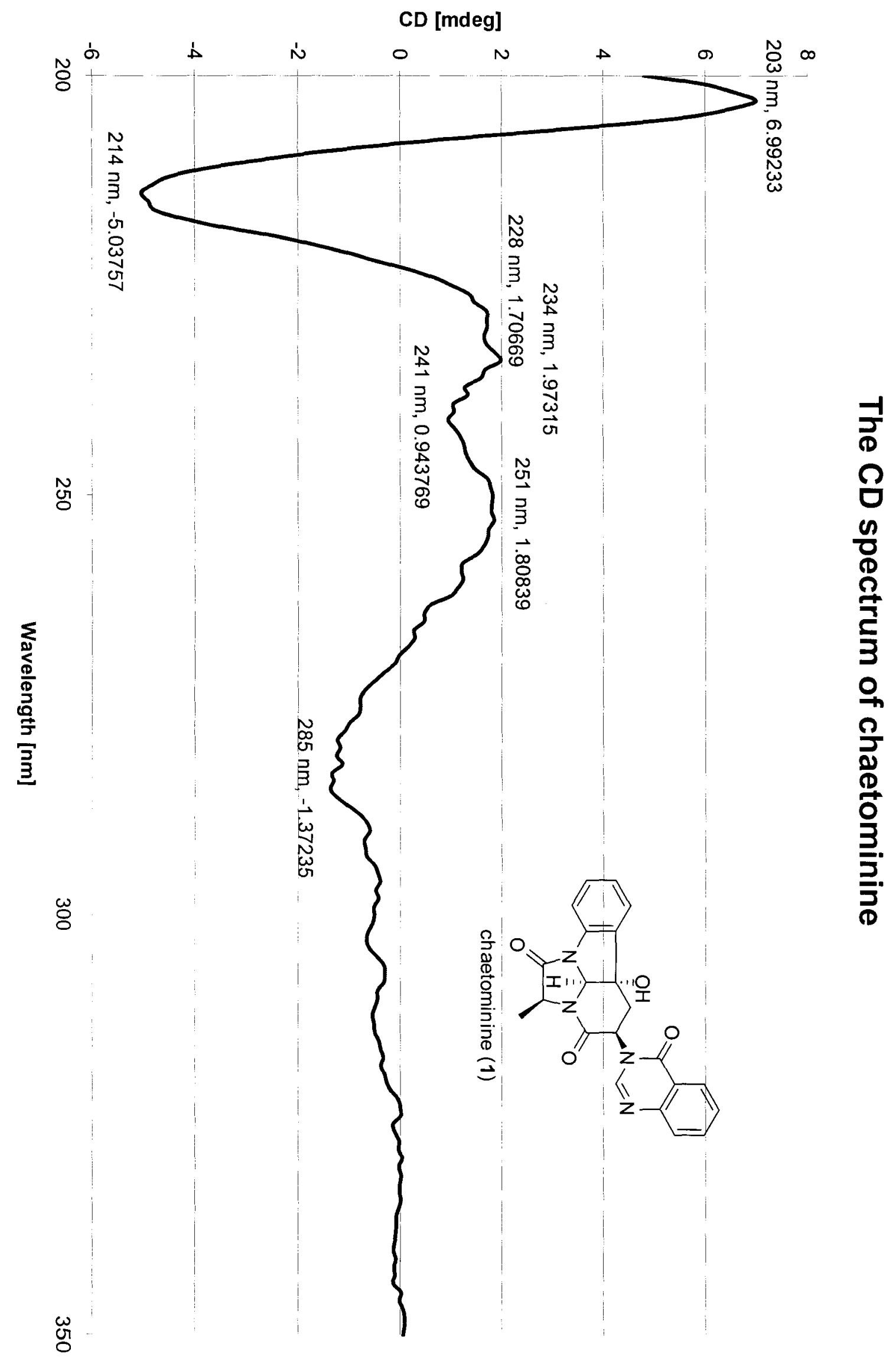


Synthesis of Chaetominine

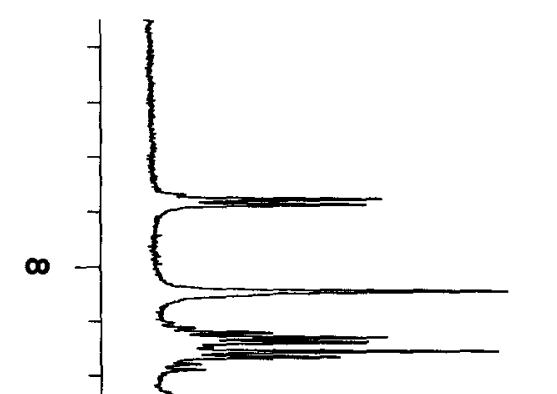

$\underline{\text { Snider and Wu}}$

$\underline{\mathrm{S} 26}$

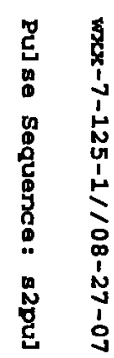

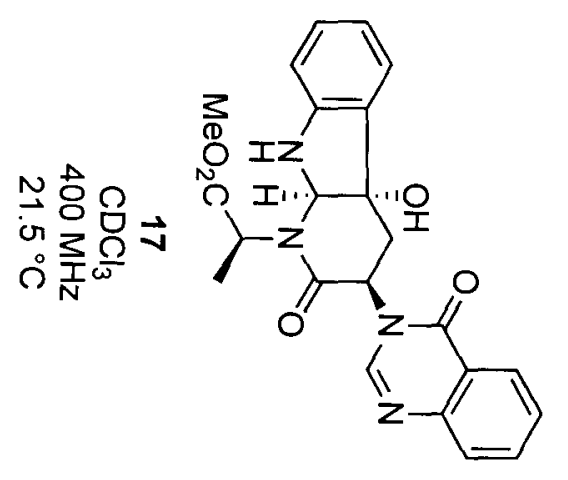

